\section{Presentation and management of paroxysmal nocturnal hemoglobinuria: a single-center experience}

\author{
Mehmet Sinan Dal, Abdullah Karakuş, \\ Mehmet Önder Ekmen, Orhan Ayyildiz \\ Department of Hematology, Dicle \\ University, Diyarbakir, Turkey
}

\section{Abstract}

Paroxysmal nocturnal hemoglobinuria (PNH) is a rare acquired disorder characterized by intravascular hemolysis. Real-world experience of PNH management is largely unreported. A retrospective analysis was undertaken based on medical records from six patients with PNH [two with aplastic anemia (AA)] treated at our center, Dicle University, Turkey. Diagnosis was based on granulocyte PNH clones, ranging from $93 \%$ to $66 \%$. All patients had symptoms consistent with PNH. One patient was managed adequately with supportive measures only. Five were treated with the complement inhibitor eculizumab. Follow-up data ( $<1$ year) were available in four cases (the fifth had received only three infusions by final follow-up). Hemoglobin level in these four patients increased from 4.1-7.2 $\mathrm{g} / \mathrm{dL}$ to $8.3-13.0 \mathrm{~g} / \mathrm{dL}$. Lactate dehydrogenase, a marker for hemolysis, decreased profoundly in the two non-AA patients, with more minor improvements in the two AA patients. Weakness and fatigue improved in all eculizumab-treated patients. Four of the five treated patients became transfusion independent, including the patient given only three infusions. In the remaining case, a patient with AA, transfusion requirement decreased, and abdominal pain and dysphagia resolved. No adverse events occurred. PNH can be successfully managed in routine practice.

\section{Introduction}

Paroxysmal nocturnal hemoglobinuria (PNH) is a rare, acquired disorder with an estimated incidence of 1.5 to 2 cases per million of the population per year. ${ }^{1}$ It can develop in the absence of any other bone marrow disorder or secondary to conditions such as aplastic anemia (AA). PNH is characterized by somatic mutations in hematopoietic stem cells. ${ }^{2}$ These mutations inhibit synthesis of the glycophosphatidylinositol (GPI) anchor, leading to a deficit of GPI-linked complement regulatory proteins on the cell surface of erythro- cytes. ${ }^{3}$ As a consequence, the erythrocytes become susceptible to uncontrolled complement-mediated intravascular hemolysis. Symptoms include anemia, dyspnea, fatigue, headache, dysphagia, erectile dysfunction and abdominal pain, and quality of life is compromised. Rates of hospitalization for PNH-related complications such as renal dysfunction and pulmonary hypertension are high, ${ }^{4}$ and approximately $25 \%$ of patients die within 10 years of diagnosis, ${ }^{5}$ most frequently due to thromboembolic events. ${ }^{4}$

Allogeneic stem cell transplantation offers a potential cure but the associated morbidity and risk of mortality usually precludes its use in cases of PNH. Instead, the management of PNH has historically been based on supportive measures, with frequent use of red blood cell transfusions and anticoagulation therapy. ${ }^{4}$ Since 2007, the humanized monoclonal antibody eculizumab has been licensed for the treatment of PNH. It acts by binding to complement protein $\mathrm{C} 5$, thereby inhibiting terminal complement formation, ${ }^{6}$ and in clinical trials has significantly reduced hemolysis, transfusion requirements, the incidence of thrombotic events and improved fatigue and quality of life, with an acceptable safety profile. . $^{711}$

The rarity of PNH means that reports of its presentation and treatment outside the confines of a clinical trial, or real-world experience with eculizumab therapy in this setting, are extremely sparse. ${ }^{12}$

We report here six cases of PNH who were identified and managed at our center under routine conditions.

\section{Materials and Methods}

The Department of Hematology of Dicle University in southeast Turkey is the specialist hematology referral center for a population of approximately six million people. All patients presenting with Coombs-negative nonimmune hemolytic anemia, refractory anemia myelodysplastic syndrome, AA or atypicallylocated thrombosis are screened for PNH. Patient records from January 2011 to November 2015 were reviewed to identify all cases of PNH.

Diagnostic procedures were performed according to the International Clinical Cytometry Society guidelines. ${ }^{13}$ Diagnosis of PNH requires that at least two different GPI protein deficiencies be detected within two different cell lines from granulocytes, monocytes or erythrocytes. At our center we used fluorescently-labeled aerolysin (FLAER) flow cytometry, the most sensitive and reliable diagnostic method available, to assess GPI protein deficiency in granulocytes and monocytes since previous transfusions or massive hemolysis
Correspondence: Mehmet Sinan Dal, Department of Hematology, Faculty of Medicine, Dicle University, 21280 Yenişehir, Diyarbakır, Turkey. Tel.: $+90.4122 .488001 / 4921$ - Fax: +90.4122 .488440 . E-mail: dr.sinandal@gmail.com

Key words: Hemolysis; aplastic anemia; paroxysmal nocturnal hemoglobinuria; eculizumab.

Contributions: MSD collected and analyzed the data, and undertook the manuscript development; AK and MÖE followed up the patients; OA critically reviewed and edited the manuscript.

Conflict of interest: the authors declare no potential conflict of interest.

Funding: there was no external funding. Editorial support from a freelance medical writer was funded by Alexion Pharma GmbH, Zurich, Switzerland.

Received for publication: 12 January 2016.

Revision received: 4 March 2016.

Accepted for publication: 8 March 2016.

This work is licensed under a Creative Commons Attribution-NonCommercial 4.0 International License (CC BY-NC 4.0)

CC Copyright M. Sinan Dal et al., 2016

Licensee PAGEPress, Italy

Hematology Reports 2016; 8:6409

doi:10.4081/hr.2016.6409

can bias data from erythrocytes.

Autoimmune hemolytic anemia was ruled out by the direct Coombs test. Diagnostic bone marrow aspiration and biopsy were performed in cases of bone marrow failure.

Red blood cell transfusions were given to patients in whom hemoglobin $(\mathrm{Hb})$ level was $<7 \mathrm{~g} / \mathrm{dL}$ or in whom symptoms of anemia were present. Thrombocyte transfusions were given if thrombocyte level decreased below 20.000 $\mathrm{mm}^{3}$ or if bleeding occurred in patients with AA. In patients with a confirmed diagnosis of $\mathrm{PNH}$, eculizumab was initiated in response to presentation with fatigue, with or without other symptoms, in transfusion-dependent patients. Eculizumab was administered by intravenous infusions, dosed as per the manufacturer's recommendations i.e. $600 \mathrm{mg}$ weekly for the first four weeks, followed by a dose of $900 \mathrm{mg}$ one week later, and $900 \mathrm{mg}$ every two weeks thereafter. ${ }^{14}$ Patients were assessed prior to the start of eculizumab therapy and every two weeks thereafter, including complete blood count and measurement of lactate dehydrogenase (LDH), indirect bilirubin, creatinine and reticulocytes to evaluate the extent of hemolysis. FLAER testing was repeated every six months to evaluate clone size.

Due to the increased risk of Neisseria 
meningitidis meningococcal infection in patients treated with eculizumab, ${ }^{15}$ all patients treated with eculizumab were vaccinated with a polyvalent meningococcal vaccine. Patients were closely monitored for early signs and symptoms of meningococcal infection.

Data collection from the medical records included demographics at the time of diagnosis, presence or absence of AA or other bone marrow conditions, clinical presentation, transfusion requirements, use of medication to treat $\mathrm{PNH}$ or AA, and the dose and number of eculizumab infusions (if used). Information on PNH clone sizes in both granulocytes and monocytes was collected but data from granulocytes were used to establish clone sizes. Hematology and biochemistry results were captured at the time of diagnosis and at the last follow-up point prior to analysis in November 2015.

\section{Results}

In total, approximately 600 patients were screened for PNH during January 2011 to September 2015. Small asymptomatic PNH clones were detected in 14 patients, ranging in size from $0.1 \%$ to $12 \%$. A diagnosis of symptomatic PNH was confirmed in six cases, which are described here. Last follow-up of these six patients took place in November 2015.

The six patients with $\mathrm{PNH}$ ranged from 17 to 46 years of age (Table 1). Five were male, one was female. In two patients with bone marrow failure, the presence of AA was confirmed by bone marrow aspiration and biopsy (Patients \#3 and \#4). All six patients presented with fatigue, with or without other symptoms typical of PNH (Table 1). No patient had any comorbid disease and there was no evidence of organomegaly. No patient had experienced PNH-related complications, including thrombosis. Granulocyte PNH clone sizes ranged from $93 \%$ to $66 \%$, and were generally mirrored by monocyte clone sizes (Table 1). The highest granulocyte clone sizes were seen in three of the non-AA patients (Patients \#1, \#2 and \#6). All patients had a negative direct Coombs test.

All patients were severely anemic (range 4.1-7.2 $\mathrm{g} / \mathrm{dL}$ ), particularly the two patients with AA (4.1 and $4.8 \mathrm{~g} / \mathrm{dL}$ ). Mean corpuscular volume (MCV) values at baseline were within or above the normal reference range. Counts of white blood cell counts, neutrophils and platelets were low in the patients with AA, as expected. Levels of LDH, a marker for hemolysis, were dramatically elevated in three of the patients with PNH and no AA (Patient \#1, 1687 IU/L; Patient \#2, 1995 IU/L; Patient \#6, 1136 IU/L), less severely raised in the other non-AA patient (706 IU/L), mildly elevated in one of the patients with AA (464 IU/L) and within nor- mal range for the second patient with AA (262 IU/L), consistent with the observed differences in granulocyte clone sizes. Compatible with this, reticulocyte counts and bilirubin levels were higher in the patients without AA, indicative of high erythrocyte turnover. Both of the patients with $\mathrm{PNH}$ and $\mathrm{AA}$ were receiving immunosuppressive treatment with horse antithymocyte globulin and cyclosporine at presentation (Patients \#3 and \#4). Two patients, one without AA (Patient \#2) and one with AA (Patient \#4), were receiving steroid therapy. In both patients, cyclosporine therapy was ongoing at last follow-up, after a minimum of two years' treatment.

All six patients were offered eculizumab. One patient (Patient \#6) who had Hb $11 \mathrm{~g} / \mathrm{dL}$ and only moderately increased LDH (706 IU/L), was offered eculizumab to reduce the risk of thrombotic complications. He declined, and was instead managed using red blood cell transfusions, with short-term steroid therapy during hemolytic attacks. At last follow-up, more than four years after diagnosis, he remains free of PNH-related complications but continues to experience fatigue.

Four patients received eculizumab as planned, being given a total of $8,18,25$ and 23 doses during the analysis period, respectively, with a duration of $11,31,47$ and 41 weeks between start of therapy and last follow-up. A further patient (Patient \#5) had received only

Table 1. Patient characteristics.

\begin{tabular}{lcccccc} 
& Patient \#1 & Patient \#2 & Patient \#3 & Patient \#4 & Patient \#5 & Patient \#6 \\
Age, years & 46 & 24 & 31 & 17 & 45 & 30 \\
Gender & Male & Male & Female & Male & Male & Male \\
\hline Symptoms of PNH & Fatigue, dyspnea & Fatigue & Fatigue, abdominal pain, dysphagia & Fatigue, easily tired & Fatigue, dark urine & Fatigue \\
Date of diagnosis* & June 2015 & March 2015 & July 2014 & September 2013 & January 2011 & September 2015 \\
\hline Diagnosis & PNH & PNH & PNH, aplastic anemia & PNH, aplastic anemia & PNH & PNH \\
PNH clone size, ${ }^{\circ}$ granulocytes (CD24), \% & 91 & 93 & 84 & 66 & 75 & 93 \\
\hline PNH clone size, ${ }^{\circ}$ monocytes (CD14), \% & 89 & 88 & 78 & 61 & 77 & 91 \\
\hline
\end{tabular}

PNH, paroxysmal nocturnal hemoglobinuria. *Last follow-up November 2015. ${ }^{\circ}$ Fluorescently labeled aerolysin assay.

Table 2. Hematology and biochemistry values before eculizumab treatment (baseline)\# and at last follow-up.

\begin{tabular}{|c|c|c|c|c|c|c|c|c|c|c|c|c|c|}
\hline & \multicolumn{2}{|c|}{ Patient \#1 } & \multicolumn{2}{|c|}{ Patient \#2 } & \multicolumn{2}{|c|}{ Patient \#3 } & \multicolumn{2}{|c|}{ Patient \#4 } & \multicolumn{2}{|c|}{ Patient \#5 } & \multicolumn{2}{|c|}{ Patient \#6 } & \multirow[t]{2}{*}{ Normal range } \\
\hline & B. & L. & B. & L. & B. & L. & B. & L. & B. & L. & B. & L. & \\
\hline $\mathrm{Hb}, \mathrm{g} / \mathrm{dL}$ & 7.2 & 10.6 & 6.2 & 12.0 & 4.8 & 8.3 & 4.1 & 13.0 & 11.0 & - & 7.2 & - & 14-18 (M), 12-16 (F)* \\
\hline White blood cell count, $/ \mathrm{mm}^{3}$ & 12,800 & 8800 & 8100 & 5900 & 2700 & 2600 & 1100 & 5600 & 2400 & - & 7900 & - & $5000-10,000 *$ \\
\hline Absolute neutrophil count, $/ \mathrm{mm}^{3}$ & 8200 & 4800 & 5700 & 3400 & 480 & 700 & 850 & 1280 & 900 & - & 4800 & - & $1500-8000 *$ \\
\hline Platelet count, $\times 10^{3 / \mathrm{mm}^{3}}$ & 727 & 546 & 99 & 125 & 22 & 14 & 14 & 21 & 70 & - & 295 & - & $200-500^{*}$ \\
\hline Reticulocytes, \% RBC & 11 & 3.5 & 4.4 & 2 & 1.1 & 1 & 0.8 & 0.9 & 5.6 & - & 5.1 & - & $0-1 \% *$ \\
\hline Lactate dehydrogenase, IU/L & 1687 & 233 & 1995 & 216 & 464 & 204 & 262 & 238 & 706 & - & 1136 & - & $105-333^{\circ}$ \\
\hline Total bilirubin, mg/dL & 2.2 & 3.0 & 2.8 & 2.3 & 1.1 & 1.1 & 0.6 & 0.5 & 2.2 & - & 0.7 & - & $<1.0^{*}$ \\
\hline Direct bilirubin, mg/dL & 0.7 & 0.8 & 0.7 & 0.6 & 0.5 & 0.3 & 0.2 & 0.2 & 0.6 & - & 0.4 & - & $<0.4^{*}$ \\
\hline
\end{tabular}

"Values recorded at clinic visit immediately prior to start of eculizumab therapy . B, baseline; L, last follow-up; Hb, hemoglobin; RBC, red blood cells. M, male; F, female. *Food and Drug Administration Investigations Operations Manual 2015; US National Library of Medicine. Time from start of eculizumab therapy to last follow-up was 11,31, 47 and 41 weeks in Patients \#1, \#2, \#3 and \#4, respectively. Follow-up data were not available for Patient \#5 due to short follow-up. Patient \#6 declined eculizumab therapy. 
three doses of eculizumab therapy by the end of the follow-up period, such that meaningful post-treatment laboratory results were not available. All patients remained on eculizumab treatment at last follow-up. In each case, symptoms of fatigue and weakness resolved. Four of the five treated patients became transfusionindependent, including the patient who had received only three doses, and the fifth patient (who had AA) showed a reduced requirement for transfusion. No PNH-related symptoms have been reported during eculizumab therapy. Each patient showed a pronounced increase in Hb levels after starting eculizum$\mathrm{ab}$, although none reached the normal range (Table 2). Hb at last follow-up was lowest in Patient \#3 (8.3 g/dL), who had severe AA, but abdominal pain and dysphagia stopped completely after starting eculizumab therapy. Allogeneic hematopoietic stem cell transplantation was planned from an HLA-matched sibling donor for this patient but she refused the procedure. Patient \#4, who also had AA, had been receiving immunosuppression for a year prior to the start of eculizumab but $\mathrm{Hb}$ increased from $4.1 \mathrm{~g} / \mathrm{dL}$ at baseline to $13.0 \mathrm{~g} / \mathrm{dL}$ at last follow-up despite LDH in the normal range when eculizumab was started. The physiological basis for this is unclear.

The two non-AA patients in whom follow-up data were available (Patients \#1 and \#2) both showed a decrease from highly elevated levels of $\mathrm{LDH}$ to normal levels, indicating a profound reduction in hemolysis. LDH declined to within normal range in one patient with AA (Patient \#3), and remained in normal range in the second patient with AA (Patient \#4).

No thrombotic episodes or other PNH-related complications were observed in any patient during follow-up, and there were no cases of $N$. meningitidis infection.

There were no serious adverse events associated with eculizumab therapy. During followup, one patient experienced an upper respiratory tract infection (Cryptic tonsillitis) and another patient developed herpetic conjunctivitis, both of which were treated successfully with penicillin and acyclovir.

\section{Discussion}

The six patients with PNH described here had relatively severe disease compared to the worldwide PNH population ${ }^{4}$, as indicated by granulocyte clone size, LDH levels and clinical symptoms. The patients without AA generally had higher clone sizes than the two patients with $\mathrm{AA}$, as reported in the literature, ${ }^{4}$ and higher levels of LDH. This indicates the dominance of hemolysis as the driver for anemia and clinical symptoms in these non-AA individuals. In the two patients for whom PNH was secondary to AA, LDH levels were only moderately above normal or within normal range.

Symptoms of PNH, most notably fatigue and weakness, entirely resolved under eculizumab. Follow-up data from two non-AA patients given eculizumab therapy demonstrated a dramatic decline in elevated LDH levels, reaching the normal range. In the two patients with $\mathrm{PNH}$ secondary to AA, hemolysis was less pronounced prior to treatment, but addition of eculizumab to long-term immunosuppressive therapy was nevertheless associated with $\mathrm{Hb}$ increases of 3.5 and $8.9 \mathrm{~g} / \mathrm{dL}$, respectively. No patient experienced a thrombotic event or developed renal dysfunction during eculizumab therapy, and no adverse events occurred.

Dosing of eculizumab according to the manufacturer's recommendations was practicable under routine conditions. At final follow-up, all patients remained on eculizumab therapy at the full recommended dose. One patient, aged 17 years and weighing $80 \mathrm{~kg}$, received the standard adult dose instead of the recommended pediatric dose (for patients $<18$ years) due to his height and weight.

Both the patients with $\mathrm{AA}$ were receiving immunosuppression with horse antithymocyte globulin and cyclosporine at the point when PNH was diagnosed. This regimen achieves response rates of $50-60 \%$ even in severe $\mathrm{AA},{ }^{16,17}$ but in these two patients the baseline $\mathrm{Hb}$ remained below $5 \mathrm{~g} / \mathrm{dL}$ despite ongoing immunosuppressive therapy. Immunosuppression does not affect PNH clone size, ${ }^{18}$ and here the clone sizes $(>50 \%)$ indicated a need for eculizumab to minimize the risk for PNH-associated complications. ${ }^{19}$ Case reports of eculizumab therapy in $\mathrm{PNH}$ with AA are rare. Hill et al. described a 48-year-old transfusion-dependent male who developed AA and was diagnosed with PNH five years later. ${ }^{20}$ In the absence of immunosuppressive therapy, six months' treatment with eculizumab reduced hemolysis and lowered the need for transfusions by $44 \%$, with stable $\mathrm{Hb}^{20}$ Asano and colleagues have reported the case of a 65 year-old woman in whom a hemolytic attack, triggered by sepsis, was diagnosed as PNH with AA. ${ }^{21}$ Eculizumab therapy successfully controlled the hemolytic attack, after which horse antithymocyte globulin and cyclosporine were introduced. The patient was able to stop blood transfusions and there was no recurrence of hemolysis. In the two patients with $\mathrm{PNH}$ and $\mathrm{AA}$ in our series, Hb improved markedly but in one case (Patient \#4) baseline $\mathrm{LDH}$ was in the normal range and it is not clear what drove the improvement in $\mathrm{Hb}$. He had been receiving immunosuppressive therapy for a year, so this is not likely to account for the changes observed after starting eculizumab. Further research is required to elucidate the effects of eculizumab in this setting.

The rarity of PNH means that even special- ist referral centers see few cases. In this series of six patients, diagnosis was readily established based on FLAER analysis of granulocytes. Despite the relative severity of PNH in our series, symptoms resolved and elevated LDH levels normalized following eculizumabmediated inhibition of complement-mediated intravascular hemolysis. Eculizumab was administered successfully outside the confines of a clinical trial with good tolerability. Although no patient was followed for more than a year under eculizumab, long-term data from clinical trials (up to 5.5 years) suggest that the benefits of eculizumab are sustained. ${ }^{22-24}$

\section{References}

1. Sahin F, Ozkan MC, Mete NG, et al. Multidisciplinary clinical management of paroxysmal nocturnal hemoglobinuria. Am J Blood Res 2015;5:1-9.

2. Takeda J, Miyata T, Kawagoe $\mathrm{K}$ et al. Deficiency of the GPI anchor caused by a somatic mutation of the PIG-A gene in paroxysmal nocturnal hemoglobinuria. Cell 1993;73:703-11.

3. Kinoshita T, Ohishi K, Takeda J. GPIanchor synthesis in mammalian cells: genes, their products, and a deficiency. J Biochem 1997;122:251-7.

4. Schrezenmeier H, Muus $P$, Socié G, et al. Baseline characteristics and disease burden in patients in the International Paroxysmal Nocturnal Hemoglobinuria Registry. Haematologica 2014;99:922-9.

5. de Latour RP, Mary JY, Salanoubat C, et al. Paroxysmal nocturnal hemoglobinuria: natural history of disease subcategories. Blood 2008;112:3099-106.

6. Thomas TC, Rollins SA, Rother RP, et al. Inhibition of complement activity by humanized anti-C5 antibody and singlechain Fv. Mol Immunol 1996;33:1389-401.

7. Hillmen P, Hall C, Marsh JC, et al. Effect of eculizumab on hemolysis and transfusion requirements in patients with paroxysmal nocturnal hemoglobinuria. N Engl J Med 2004;350:552-9.

8. Brodsky RA, Young RS, Antonioloi E, et al. Multicenter phase 3 study of the complement inhibitor eculizumab for the treatment of patients with paroxysmal nocturnal hemoglobinuria. Blood 2008;111:18407.

9. Kanakura Y, Ohyashiki I, Shichishima T, et al. Safety and efficacy of the terminal complement inhibitor eculizumab in Japanese patients with paroxysmal nocturnal hemoglobinuria: the AEGIS clinical trial. Int $\mathrm{J}$ Hematol 2011;93:36-46. 
10. Reis UM, Schwartz J, Sakamoto KM, et al. Efficacy and safety of eculizumab in children and adolescents with paroxysmal nocturnal hemoglobinuria. Pediatr Blood Cancer 2014;61:1544-50.

11. Hillmen P, Young NS, Schubert J, et al. The complement inhibitor eculizumab in paroxysmal nocturnal hemoglobinuria. $\mathrm{N}$ Engl J Med 2006;355:1233-43.

12. Kelly RJ, Hill A, Arnold LM, et al. Long-term eculizumab in paroxysmal nocturnal hemoglobulinuria: sustained efficacy and improved survival. Blood 2011;117:678692.

13. Borowitz MJ, Craig FE, Digiuseppe JA, et al. Guidelines for the diagnosis and monitoring of paroxysmal nocturnal hemoglobinuria and related disorders by flow cytometry. Cytometry B Clin Cytom 2010;78:211-30.

14. Alexion Pharmaceuticals Inc,. Soliris ${ }^{\circledR}$ Prescribing Information. 2007. Available from: http://soliris.net/resources/ pdf/ soliris_pi.pdf

15. McKeage K. Eculizumab: a review of its use in paroxysmal nocturnal haemoglobinuria. Drugs 2011;71:2327-45.

16. Frickhofen N, Kaltwasser JP, Schrezenmeier $\mathrm{H}$, et al. Treatment of aplastic anemia with antilymphocyte globulin and methylprednisolone with or without cyclosporine. The German Aplastic Anemia Study Group. N Engl J Med 1991;324:1297-304.

17. Scheinberg $\mathrm{P}, \mathrm{Wu} \mathrm{CO}$, Nunez $\mathrm{O}$, et al. Treatment of severe aplastic anemia with a combination of horse antithymocyte globulin and cyclosporine, with or without sirolimus: a prospective randomized study. Haematologica 2009;94:348-54.

18. Parker CJ. Paroxysmal nocturnal hemoglobinuria. Curr Opin Hematol 2012;19:141-8.

19. Scheinberg P, Marte M, Nunez 0, Young NS. Paroxysmal nocturnal hemoglobinuria clones in severe aplastic anemia patients treated with horse anti-thymocyte globulin plus cyclosporine. Haematologica 2010; 95:1075-80.

20. Hill A, Richard SJ, Rother RP, Hillmen P. Erythopoietin treatment during comple- ment inhibition with eculizumab in a patient with paroxysmal nocturnal hemoglobinuria. Haematologica 2007;92:e31-3.

21. Asano J, Ueda R, Tanaka Y, et al. Effects of immunosuppressive therapy in a patient with aplastic anemia-paroxysmal nocturnal hemoglobinuria (AA-PNH) syndrome during ongoing eculizumab treatment. Intern Med 2014;53:125-8.

22. Hill A, Hillmen P, Richards SJ, et al. Sustained response and long-term safety of eculizumab in paroxysmal nocturnal hemoglobinuria. Blood 2005;106:2559-65.

23. Hillmen P, Muus P, Röth A, et al. Long-term safety and efficacy of sustained eculizumab treatment in patients with paroxysmal nocturnal haemoglobinuria. Br J Hematol 2013;162:62-73.

24. Kanakura Y, Ohyashiki K, Shichishima T, et al. Long-term efficacy and safety of eculizumab in Japanese patients with PNH: AEGIS trial. Int J Hematol 2013;98: 406-16. 